ÉGYPTE monde arabe

\section{Égypte/Monde arabe}

25 | 1996

Anthropologies de l'Égypte 2

\title{
Situer le village égyptien. La recherche d'une unité représentative
}

Traduit de l'anglais par Jean-Noël Ferrié

\section{Reem Saad}

\section{OpenEdition}

\section{Journals}

Édition électronique

URL : https://journals.openedition.org/ema/826

DOI : $10.4000 /$ ema.826

ISSN : 2090-7273

Éditeur

CEDEJ - Centre d'études et de documentation économiques juridiques et sociales

Édition imprimée

Date de publication : 30 mars 1996

Pagination : 41-50

ISSN : 1110-5097

Référence électronique

Reem Saad, «Situer le village égyptien. La recherche d'une unité représentative », Égypte/Monde arabe [En ligne], 25 | 1996, mis en ligne le 08 juillet 2008, consulté le 07 juillet 2022. URL : http://

journals.openedition.org/ema/826; DOI : https://doi.org/10.4000/ema.826

Ce document a été généré automatiquement le 7 juillet 2022

Tous droits réservés 


\title{
Situer le village égyptien. La recherche d'une unité représentative
}

\author{
Traduit de l'anglais par Jean-Noël Ferrié
}

\section{Reem Saad}

1 Toute étude anthropologique sur l'Égypte rurale se doit de prendre le village comme point de départ ${ }^{1}$. Aussi bien pour des raisons pratiques qu'empiriques, il est le premier " terrain ». Mais est-ce pour autant une unité d'analyse pertinente? Beaucoup de choses peuvent être dites sur l'inadéquation du "village » et du " paysan », aussi bien comme catégorie sociale que comme catégorie analytique. L'essentiel de cette argumentation est que le village fait partie d'une structure plus importante, ou d'un système allant de l'interdépendance avec la ville la plus proche à la dépendance vis-àvis du système capitaliste mondial et de la division internationale du travail.

2 De fait, la continuité entre le village égyptien et le monde extérieur ne peut être ignorée. L'adoption de technologies nouvelles et de moyens de communication modernes, l'apparition d'une jeune génération éduquée et ayant des goûts urbains, la dépendance d'un nombre croissant de villageois vis-à-vis du monde extérieur sont les signes de cette continuité. Le village égyptien n'est donc une unité clairement délimitée que dans l'imagination de certains orientalistes, de certains anthropologues traditionnels ou de membres de la classe moyenne urbaine.

3 À un autre niveau, cette unité pose des difficultés particulières à l'anthropologie et occupe, d'ailleurs, une place centrale dans les débats sur la relation problématique entre ethnographie et théorie. Ceci soulève maintes questions dans un contexte intellectuel et universitaire dominé par différents critères de validité. Je me réfère ici, tout particulièrement, à la question de la "représentativité ", nombre d'entre nous se trouvant souvent placés devant la question : comment peut-on généraliser des résultats si l'on n'a travaillé que dans un seul lieu? Dans cet article, je tente de montrer comment la notion de «voix» peut pratiquement s'appliquer au village égyptien et 
éclairer les aspects de la question de la « représentativité » qui ne portent pas sur la distribution statistique et spatiale de caractères.

La question des « voix » : qui parle pour qui?

4 Nombre d'études d'anthropologie et d'histoire égyptienne correspondent à l'intérêt récent de la recherche en sciences sociales pour le point de vue des marginaux et des groupes sociaux dominés. Ces études se penchent généralement sur la question de la résistance et des " voix ", intérêt lié à la volonté de compenser le biais élitiste des écrits historiques et politologiques facilement enclins à ignorer la «voix» des groupes sociaux dominés, comme les paysans et les femmes².

En général, les études qui se donnent comme objet de présenter le point de vue de ces groupes se trouvent confrontées au problème de trouver leurs " voix ». L'enthousiasme parfois excessif avec lequel les «sans-pouvoirs» ont été introduits dans les études scientifiques a entraîné une certaine tendance romantique à les glorifier en termes de " résistance » (Abu-Lughod, 1990). Mais l'idée de « donner une voix aux sans-voix », liée à celle de "majorité silencieuse", ne va pas de soi, principalement en raison de ses connotations condescendantes. L'idée de " voix » et la question " qui parle pour qui » est particulièrement importante pour les anthropologues, et étroitement liée à la question de la représentation dans les écrits ethnographiques.

6 À propos du débat sur la multiplicité des " voix " impliquées par la description, Appadurai (1987) remarque que ce ne sont pas seulement les anthropologues qui «parlent pour» les indigènes, mais aussi... «nos informateurs qui sont souvent en train de parler pour nous ». C'est en insistant ouvertement sur ce dernier point que Lila Abu-Lughod semble se dissocier de la prétention de « donner une voix aux sans-voix ». Dans Writing Women's World, qui repose principalement sur des récits autobiographiques de bédouines, L. Abu-Lughod indique clairement que, bien que la sélection de ces histoires soit d'abord guidée par l'intérêt pour la vie de ces femmes, elle a « essayé, en manipulant, reconfigurant et juxtaposant les récris des femmes et des hommes, de les faire parler particulièrement de leurs soucis comme de ce qui est un souci pour leur public ». En se nommant elle-même le " nouveau conteur » de ces récits, elle dissipe l'illusion de l'ethnographe transparent qui transmettrait purement et simplement la « voix » des autres.

7 Cependant, comme nous le rappellent les Comaroff, «l'ethnographie, en aucun cas, ne doit parler pour les autres mais sur les autres ». Et les « voix » sont un de ces concepts avec lequel nous devons travailler, même si nous sommes irrités par l'usage vulgarisé qui en est fait. Les historiens, qui sont aussi concernés par ce problème, le traitent différemment des anthropologues. Ils s'intéressent davantage à ce que disent les voix plutôt que d'en parler avec emphase ou de tenter de se les approprier pour parler à travers elles. Le problème méthodologique posé par la manière de rendre compte des "voix", des points de vue ou de la vie quotidienne de groupes qui sont exclus des rapports officiels précède logiquement le débat sur la représentativité.

8 En tout cas, il me semble qu'à ce niveau, l'intérêt de connaître le point de vue des groupes sociaux sous-représentés a été établi. Nous devons maintenant nous demander ce qu'il faut en faire. Ce ne peut être seulement pour affirmer que les déshérités ont une voix ; nous devons plutôt nous centrer sur ce qu'ils disent actuellement plutôt que de célébrer seulement l'existence de cette « voix ». Dans la partie qui suit, Je présenterai une façon d'utiliser la voix des paysans pour situer le village égyptien contemporain et pour en décrire les habitants et leurs relations avec les différentes 
sphères sociales dont ils font partie. Je discuterai la notion de voix en relation avec le problème anthropologique rémanent de la représentativité. J'en ai longuement traité lors de mon travail de thèse ${ }^{3}$, dans lequel j'ai étudié le discours subordonné des paysans en relation avec le discours officiel de la classe moyenne urbaine.

La représentativité : problèmes et implications

9 Avant d'aller plus loin, je dois mentionner que la question de la représentativité s'est posée à moi de façon particulièrement complexe en tant qu'anthropologue égyptienne travaillant en Égypte. Ce problème est apparu non seulement à propos des relations entre ethnographie et théorie, mais aussi eu égard au contexte spécifique de la scène intellectuelle égyptienne. En discutant de mon sujet de travail avec différents intellectuels et universitaires égyptiens, je fus rapidement mise en cause sous prétexte que le village d'Imam, où j'ai effectué mon travail de terrain, n'était pas forcément représentatif des «villages égyptiens" et qu'en conséquence, mes résultats ne pouvaient être généralisés.

Il y avait, je pense, deux raisons principales à cette réaction. La première tenait au fait de présenter un matériel ethnographique à une assistance indigène qui comparait en permanence son expérience personnelle (ou plutôt sa mémoire) avec mes résultats. Donnant de l'importance au «village égyptien » aussi bien comme lieu d'« authenticité » et de « moralité » que de «retard » et d'« apathie », beaucoup de mes interlocuteurs trouvaient difficile d'accepter certains de mes constats qui contredisaient les images dominantes $\mathrm{du}$ " village ». Ces images vont des pastorales nostalgiques à l'idée que les paysans sont uniformément opprimés, désemparés et apolitiques.

11 Une autre source de désaccord tenait au climat même de la recherche en Égypte. Mon travail n'était pas conforme à la conception dominante de la "recherche scientifique ", où le terme "scientifique » est largement confondu avec le terme "statistique». La situation dans laquelle Je me trouvais comme Égyptienne écrivant sur l'Égypte était ainsi liée à la situation même de l'anthropologie locale. Mais j'espère, en traitant de problèmes méthodologiques spécifiques comme celui de la représentativité, ouvrir la voie à une discussion des pratiques intellectuelles locales éloignée des propos naïfs et nationalistes qui caractérisent le débat sur «l'indigénisation » des sciences sociales (Morsy et al., 1991).

12 Je vais maintenant tenter de discuter de la façon dont la question de la représentativité est abordée, des problèmes qu'elle pose et de ses implications. Je discuterai aussi du reproche qui me fut fait de n'apporter que des réponses « crispées » et « défensives » ce qui est peut-être le cas - aux objections de mes interlocuteurs.

13 À un certain niveau, je peux justifier le fait que les paysans d'Imam sont bien représentatifs des paysans égyptiens dans la façon dont ils perçoivent l'histoire égyptienne. Cette nécessité de « justifier » et de « défendre » la capacité de la recherche ethnographique à nous informer sur une grande échelle a posé problème à de nombreux anthropologues. Lila Abu-Lughod, bien qu'elle considère cette question comme dénuée de sens, a été amenée à y répondre, défendant le fait que la communauté bédouine qu'elle a étudiée était bien représentative de tous les bédouins Awlad Ali dont elle faisait partie : «Dans la mesure où les autres bédouins se situaient à l'intérieur du même univers social et moral (univers différent de celui des Égyptiens), et que les autres communautés visitées ne différaient de celle-ci que par la qualité et la quantité des biens traditionnels ou modernes qu'elles possédaient, je crois pouvoir affirmer que cette communauté était représentative. » (1986,p.23) 
14 Nicholas Hopkins, pour sa part, a basé son livre (Agrarian Transformation in Egypt, 1987) sur des recherches ethnographiques conduites dans un seul village, Musha, en HauteÉgypte. Il affirme la représentativité de ce village en soulignant que la campagne égyptienne présente partout les mêmes structures économiques et sociales. Il insiste en même temps sur le fait qu'il existe une grande variété de villages égyptiens, rejetant la remarque de Henry Ayrout suivant laquelle «rien, en effet, ne ressemble plus à un village égyptien qu'un autre village égyptien » $(1938$, p. 112). Il note les différences de taille, de population, de situation, d'économie, de caractéristiques politiques... De sorte que, quand il en vient à dire que « Musha n'est pas typique mais que ses problèmes sont exemplaires » (1987, p. 5), nous sommes déjà prévenus contre l'idée d'homogénéité. En outre, Musha est plus grand et beaucoup plus inscrit dans les relations capitalistes que d'autres villages; pourtant, on y retrouve tous les éléments qui participent à la construction de l'Égypte rurale actuelle : mécanisation, rotation des cultures imposée par le gouvernement, problèmes d'organisation du travail. Ainsi Musha n'est-il pas «typique » en ce sens que ce village n'est pas un spécimen, mais les problèmes qui s'y posent et les processus sociaux qui s'y déroulent sont, eux, représentatifs.

Quand la question de la représentativité est posée, elle se réfère à des unités d'analyse que l'on peut qualifier d' « échantillon » ou de cas «typique ». Il faut noter tout d'abord qu'une certaine réaction à la position traditionnelle de l'anthropologie consistant à considérer les communautés comme des entités isolées et closes a abouti à une autre position, non moins extrême, principalement adoptée par les théoriciens de la dépendance, qui dissolvent ces communautés dans une "périphérie " indifférenciée. John Comaroff (1982, p. 146) défend à bon droit la nécessité d'examiner les interactions entre ces deux niveaux, surtout afin d'en définir les frontières : "iI devient nécessaire d'expliquer le processus d'interaction à l'œuvre dans ce que l'on peut nommer l'articulation dialectique entre un système local et le contexte qui l'englobe ». En fait, la façon même dont ces deux systèmes sont définis et délimités - et, par conséquent, la ligne de démarcation entre "l'interne » et «l'externe » elle-même - est inscrite dans cette dialectique.

16 Mon intention n'est pas de nier l'existence de frontières, mais de faire porter l'observation sur les conditions dans lesquelles elles sont créées. Imam est le lieu où cette observation a pu se faire mais il n'est pas pour cela une " unité d'analyse ». Définir des frontières est davantage un objectif qu'un prerequisit de la recherche. L'essentiel de mon travail consiste à comprendre comment les habitants d'Imam décrivent leurs relations avec les événements et les processus politiques et sociaux qui se déroulent à « l'extérieur » du village. C'est la façon dont ils formulent leur relation à cet « extérieur » qui met en lumière les paramètres constitutifs du village comme unité. Ceux-ci ne sont pas davantage donnés qu'ils ne correspondent à des limites fixes, quoique les limites physiques d'Imam s'inscrivent dans le paysage d'une manière qui invite à ce genre de réification.

Les gens d'Imam se définissent eux-mêmes comme paysans, membres d'une classe défavorisée. Égyptiens et habitants d'Imam. Leur appartenance au village n'est ainsi qu'une des façons par lesquelles ils se définissent. Ils insistent différemment sur l'une ou l'autre de leur identité selon les contextes mais, dans tous les cas, ils n'ont pas de doute sur leur appartenance aux divers groupes auxquels ils s'identifient et sur le fait qu'ils peuvent parler au nom des autres membres. Les exemples qui suivent illustrent comment la participation des villageois à plusieurs systèmes d'appartenance déplace le 
centre de l'analyse du village considéré comme une unité à la dynamique des interactions.

Durant mon séjour à Imam, j'ai accompagné mon hôte, Abu Sa'îd, en visite chez l'un de ses amis à Fayoum. La femme de ce dernier, une citadine, racontait une dispute qu'elle avait eue avec une autre femme. Elle fit, à un moment donné, une remarque désobligeante sur le caractère "paysan " (entendu dans le sens de rustre\} de cette dernière. Le fait qu'Abû Sa'îd prenne cette remarque personnellement et défende alors les paysans en général nous rappela qu'il appartenait à un vaste groupe - les paysans qui, pour être hétérogène et approximativement défini, n'en prenait pas moins des caractéristiques concrètes dans des situations d'interaction spécifiées.

Comme classe subordonnée, les villageois d'Imam se comparent aux autres pauvres et aux opprimés. Ils sont le « petit peuple » (al-nas al-sughayyara) tandis que l'élite est le « grand peuple " (al-nas al-kabîra). Quand ils évoquent l'oppression ou les mauvais traitements qu'ils subissent de la part de "supérieurs", ils utilisent souvent des expressions comme: "C'est ainsi que les gens comme nous sont traités ", impliquant qu'ils se reconnaissent comme appartenant à une collectivité de démunis. Dans ce cas, la représentativité n'est pas à rechercher du point de vue du village mais du point de vue de la relation entre démunis et puissants, dans une dynamique propre.

Les villageois se définissent aussi comme Égyptiens. Ils s'identifient et se déclarent comme représentants de la nation égyptienne. Cet aspect de leur identité trouve sa plus claire expression en temps de guerre ou par rapport à la migration. La principale source d'altercations entre Irakiens et travailleurs immigrés égyptiens en Irak résidait dans les insultes que les Irakiens adressaient à l'Égypte, ressenties comme des affronts dans la mesure où les travailleurs égyptiens se sentaient les représentants de leur pays. Comme le disait Abu Ashraf, un autre habitant d'Imam :

Si quelqu'un insulte l'Égypte en général, il m'insulte aussi puisque je suis Égyptien. Les anecdotes qui précèdent suffisent pour donner une indication sur le type de généralisations (ou plutôt d'abstractions) qu'on peut relever en examinant des situations spécifiques. Plutôt que d'avoir affaire à « l'Égypte rurale », nous avons affaire à l'identité paysanne, à l'appartenance de classe et à l'identité nationale.

Dans les deux cas d'Abu-Lughod et d'Hopkins cités plus haut, la défense de la représentativité est limitée au domaine d'investigation de ces auteurs : l'univers social et moral des relations entre honneur et poésie pour le premier, les éléments structuraux des transformations agraires pour le second. En ce qui concerne mon propre travail, la question de ia perception de l'histoire par les paysans implique l'étude d'un discours dominé (subordinate discourse) en relation avec le discours dominant de la classe moyenne urbaine. Dans ce cas, la question qui se pose est la suivante : les paysans d'Imam peuvent-ils " parler » pour les paysans égyptiens? Leur voix est-elle représentative? Le Popular Memory Group défend la représentativité des autobiographies et des histoires de vie en se fondant sur le fait que " leurs auteurs parlent à partir d'une position particulière dans un contexte de relations sociales caractéristiques d'une société particulière à un moment particulier de l'histoire " (1982, p. 234). En d'autres termes, le « mandat » d'une « voix » particulière dépend de la position de celui qui parie à l'intérieur de la société, et pour les habitants d'Imam, c'est leur condition de "démunis » vis-à-vis de «dominants » qui les qualifie pour parler pour ceux qui partagent leur condition. Ainsi, ce n'est jamais le caractère «typique » de l'unité retenue ni l'échantillon statistique qui détermine la validité de la 
généralisation; c'est la nature des relations entre discours dominé et discours dominant.

Ce sont les multiples identités des paysans - lesquelles ne coïncident pas avec les limites physiques du village mais les transcendent -, aussi bien que la nature de mon questionnement (mettant spécifiquement en cause les frontières spatiales) qui font que le problème de la représentativité (ou pis: de l'«unité représentative») apparaît comme non seulement dénué de sens mais encore comme hors de propos. Néanmoins, la question reste posée de savoir si le travail sur une petite échelle et en un seul lieu n'est pas le tendon d'Achille de l'anthropologue dès qu'il s'agit de généraliser ses résultats. La réponse est négative en ce qui concerne mon travail : les habitants d'Imam sont représentatifs des différents mondes auxquels leurs multiples identités se réfèrent. D'un point de vue phénoménologique, à l'instar de ce qu'il en est des bédouins d'Abu-Lughod, les participants à ces différents mondes sont situés "à l'intérieur du même univers social et moral ». D'un point de vue sociologique, les villageois d'Imam partagent avec les autres ruraux la même relation de dépendance avec la ville et l'État. Le capitalisme agraire domine la campagne égyptienne et les exigences de l'État central comme des institutions aboutit à donner des caractéristiques structurelles communes à tous les villages égyptiens. Sur ce point, la ligne de défense de Hopkins s'applique parfaitement au village d'Imam.

Cependant, il existe aussi des traits spécifiquement locaux qui font du village - comme de tout autre lieu - un lieu unique. L'histoire locale d'Imam et le système spécial d'irrigation du Fayoum, avec ses implications sur l'organisation sociale et la relation avec la bureaucratie étatique, en sont des exemples. Il est encore plus important de souligner que, s'agissant d'une recherche basée sur l'expression d'expériences individuelles, celles-ci sont, par définition, uniques. Aussi, aucune généralisation ne devra se baser sur mes abstractions, qui résument ce que les gens disent plutôt qu'elles ne rendent la particularité de leurs mots et de leurs métaphores. Comme Lila AbuLughod, je suis le «nouveau conteur » de leurs histoires.

La question de la représentativité et de la généralisation et la nécessité de les défendre est en partie induite par l'intimidation des empiristes (dans le domaine de l'échantillon représentatif, ce genre de problème ne se pose pas). La pression pour la production de résultats généralisables est peut-être aussi due aux exigences des approches basées sur la multidisciplinarité et sur les "aires culturelles " (area studies\}. Ce type de pression provient en général de l'extérieur de l'anthropologie. La faiblesse de la position de cette discipline, comme celle de l'histoire-ce sont l'une et l'autre des disciplines idiographiques - tient à ce qu'elles ont tendance à être utilisées par des disciplines nomothétiques. Avant d'être aussi enthousiastes vis-à-vis de la multidisciplinarité, nous devrions d'abord voir quelle est la politique de la division des tâches promue par cette approche. Les termes et les conditions de cette " coopération » sont donnés par l'économie politique et par la sociologie, lesquelles voudraient être les disciplines intellectuelles supérieures, élaborant des théories, alors que l'histoire et l'anthropologie se chargeraient des recherches empiriques et des données nécessaires à cette élaboration. Ainsi, la pression pour que nous présentions notre matériel dans une forme "utilisable» (précisant l'étendue de son applicabilité) n'est peut-être pas très différente de ce que Michael Gilsenan notait à propos de l'anthropologie du Monde arabe, Middle East, dans les années soixante: "L'utilisation de conventions monographiques immédiatement reconnaissables, en ce qui concerne l'organisation 
des travaux, le langage utilisé et les références, tenait à la volonté positiviste de constituer un fond de 'matériaux'. » (1990, p. 223).

Conclusion

Le « village » n'est ni une entité close et isolée, ni une unité d'analyse ; il est néanmoins doté d'une importance et d'une présence sous-déterminée par le point de vue totalisant de l'économie politique. C'est une notion multiréférentietle, principalement spatiale, même si son contenu «localisateur » ne conditionne pas ses autres sens. J'ai tenté de montrer que la "signification » du village apparaissait dans ce que ses habitants disaient de lui. Il faut ainsi rechercher ses frontières dans le lien unissant « localité » et " voix ", et nous focaliser sur ses caractéristiques discursives aussi bien que sur ses caractéristiques spatiales ne peut que nous aider à saisir ses différentes dimensions. C'est la nature des " voix », bien plus que le lieu d'où les gens parient, qui indique les frontières d'une localité et définit l'intérieur et l'extérieur davantage que n'importe quel critère structurel ou physique. Comme les paysans d'Imam, je considère que le village a une frontière, mais qu'il s'agit d'une sorte de frontière provisoire et changeante.

\section{BIBLIOGRAPHIE}

Abu-Lughod L, Veiled Sentiments, University of California Press, Berkeley, 1986.

- « The Romance of Résistance: Tracing Transformations of Power through Bedouin Women », American Ethnologist n 17 (1), 1990, p. 41-45.

- Writing Women's Worlds, University of California Press, Berkeley, 1993.

Appadurai A., «Introduction: Place and Voice in Anthropological Theory », Cultural Anthropology 3 (1), 1988, p. 16-20.

Ayrout H. H., Mours et coutumes des Fellahs (trad. de The Egyptian Peasant par J. A. Villiams, Beacon Press, Boston, 1938), Payot, Paris, 1963.

Brown N., Peasant Politics in Modem Egypt, Yale University, New Haven \& Londres, 1990.

Comaroff J., « Dialectical Systems, History and Anthropology: Units of Study and Questions of theory ", Journal of Southern AfricanStudies, n8 (2), 1982.

Comaroff J. \& J, Ethnography and the Historical Imagination, Westview Press, Boulder, San Francisco, Oxford, 1992.

Gilsenan M., « Very Like a Camel: The Appearance of an Anthropologist's Middie East », Localizing Strategies (R. Pardon éd.), Scottish Academic Press, Edinbourg \& Smithsonian Institution Press, Washington, 1990.

Hopkins N., Agrarian Transformation in Egypt, Westview Press, Boulders, Londres, 1987.

Kazemi, Farhad \& Waterbury (eds), Peasants and Politics in the Modern Middle East, Florida International University Press, Miami, 1991. 
Morsy S., Nelson C., Saad R., Sholkamy H., « Anthropology and the Call for Indigenization of the Social Science in the Arab World », The Contemporary Study of the Middle East, American University of Cairo Press, Le Caire, 1991.

\section{NOTES}

1. Je voudrais exprimer ma gratitude au St. Anne's College d'Oxford pour l'aide qu'il m'a apportée durant la rédaction de cet article.

2. A ce sujet, voir (bibliographie ci-après) abu-Lughod (1993), Brown (1990), Kazemi \& Waterbury (1991).

3. Voir à ce sujet ma thèse sur La perception de l'histoire récente de l'Égypte par les paysans. J'ai conduit mon travail de terrain dans un hameau du gouvernorat du Fayoum que je désigne, ici, par le pseudonyme de "Imam ». Imam a un peu plus de 800 habitants. La plus grande partie de mon travail a eu lieu en 1989 ; par la suite, j'y ai fait plusieurs séjours de courte durée, le dernier datant de mars 1995.

\section{INDEX}

Mots-clés : anthropologie, village, fellah, terrain

\section{AUTEUR}

REEM SAAD

Université américaine du Caire 